\title{
PROBLEMAS EN EL RECONOCIMIENTO DE LAS FUNCIONES SIGNIFICATIVAS DE LAS FORMAS CANTÉ Y CANTABA EN EL DISCURSO INACTUAL DE APRENDIENTES DE ELE COREANOS
}

\author{
María Victoria SOUlÉ \\ Cyprus University of Technology
}

\section{RESUMEN}

En este estudio nos proponemos analizar los problemas más significativos que encuentran los aprendientes de ELE coreanos al utilizar las formas canté y cantaba para narrar hechos pasados. A modo de ilustración se presentan los datos obtenidos en una prueba de comprensión lectora y expresión escrita aplicada a 60 estudiantes universitarios coreanos. Los resultados revelan que no todos los contextos resultan igualmente complejos para estos aprendientes. Al mismo tiempo, se observa que mientras algunas confusiones tienden a desaparecer a medida que avanza el nivel de ELE de los aprendientes, otras parecen haberse fosilizado en la interlengua de los participantes estudiados. Entre los factores que originan este proceso se evalúa la influencia de la lengua materna junto con la instrucción recibida por los aprendientes.

Palabras Clave: Pretérito perfecto simple y pretérito imperfecto de indicativo, Discurso inactual, Enseñanza del español como lengua extranjera, Aprendientes de ELE coreanos.

\section{ABSTRACT}

The aim of this study is to analyse the main problems that South Korean learners of Spanish L2 face when using the canté and cantaba forms in order to narrate past events. Data elicited through a reading and writing test among 60 South Korean university students show that discourse context plays an important role in determining the grade of difficulty in the selection of the aforementioned verbal forms. The results also reveal that while some difficulties tend to disappear as learners progress in the knowledge of the target language, 
other errors seem to remain in the interlanguage of the participants. The influence of the learners' L1 along with the instructional treatment is examined as factors causing this phenomenon.

KeY words: Preterit and Imperfect tenses, Story Mode Narrative, Teaching Spanish as Foreign Language, Korean Learners of Spanish as a Foreign Language.

\section{INTRODUCCIÓN}

Entre los problemas gramaticales de la enseñanza del español como lengua extranjera ocupa un lugar destacado el estudio del aprendizaje de las formas canté y cantaba. Este interés no es aleatorio, ya que no son pocas las lenguas que no establecen una distinción morfológica en sus formas de pasado del modo en que lo hace la lengua española, entre esas lenguas debemos destacar la lengua coreana (Choo y Kwak, 2008). Es, precisamente, la distancia existente entre el sistema verbal español y el coreano la que ha llevado a los pocos estudios realizados hasta este momento a analizar las dificultades que entraña la distinción pretérito perfecto simple/ pretérito imperfecto de indicativo para los aprendientes coreanos. Así por ejemplo, Lee y Son (2009) defienden que, al no existir en la lengua coreana una oposición aspectual como la que encierran las formas canté y cantaba, los aprendientes no terminan de asimilar el funcionamiento de estas dos formas:

Los estudiantes coreanos se enfrentan también a una dificultad a la hora de distinguir correctamente los usos aspectuales de las formas verbales de pretérito indefinido (i.e. pretérito perfecto simple) y de pretérito imperfecto, debido a la ausencia de distinción aspectual entre perfecto e imperfecto en el tiempo pasado del coreano. (2009:9)

En esta línea se encuentra la investigación realizada por Long y Whatley (2014) en la que se afirma que el comportamiento de los aprendientes coreanos en el uso de los pretéritos perfecto simple e imperfecto de indicativo es similar al de los aprendientes de ELE anglohablantes debido a la inexistencia de una distinción aspectual, como la de las formas canté y cantaba, en la lengua materna de ambos grupos. Sin embargo, creemos que es precisamente una interpretación en términos aspectualistas la que no permite analizar cuáles son los verdaderos problemas que representa el aprendizaje del funcionamiento de los dos pretéritos, aprendizaje que en no pocas ocasiones se ve influenciado por una didáctica basada en descripciones insuficientes y poco rigurosas.

Sin rechazar la influencia de la lengua materna, en este trabajo, que se inscribe en una investigación más amplia, nos proponemos, en primer lugar, examinar cuál es el tratamiento que reciben los pretéritos perfecto simple e imperfecto de indicativo en los materiales didácticos de ELE, especialmente en aquellos materiales creados para los aprendientes coreanos. En segundo lugar, presentamos un enfoque, que creemos supera los tratamientos tradicionales que se han venido desarrollando sobre este aspecto de la gramática. Por último, con el propósito de estudiar el impacto de estas descripciones en la producción escrita de aprendientes coreanos, exponemos los resultados de un estudio en el que analizamos cuáles son las funciones significativas de las formas canté y cantaba que les resultan más complejas de adquirir. 


\section{LA DIDÁCTICA DE LAS FORMAS CANTÉ Y CANTABA}

La didáctica de las formas canté y cantaba se ha realizado desde numerosas aproximaciones. El Plan curricular del Instituto Cervantes (2006) establece que la enseñanza de los pretéritos perfecto simple e imperfecto de indicativo comienza en los niveles A1-A2 y se extiende de forma progresiva hasta los niveles $\mathrm{C} 1-\mathrm{C} 2{ }^{1}$. Los descriptores presentados en este compendio son adoptados por varios manuales y gramáticas de ELE junto a otras interpretaciones, entre las que se suele recurrir a la categoría de aspecto gramatical. Desde esta categoría la forma canté se interpreta como un tiempo perfectivo y la forma cantaba como un tiempo imperfectivo ${ }^{2}$. Esta interpretación puede observarse en las siguientes definiciones recogidas de manuales de ELE:

(1) a. "Con el indefinido se expresa una acción puntual, concreta." (Gramática práctica del español: A1-A2, 2005:122).

b. "El pretérito indefinido puede usarse para acciones que se repiten." (Uso de la gramática española: intermedio, 2006:8).

c. "El pretérito imperfecto expresa acciones repetidas en el pasado." (Uso de la gramática española: elemental, 2006:115).

d. "Se usa el pretérito imperfecto para hablar de una acción o situación en desarrollo en el pasado." (Gramática del uso del español: A1-A2, 2010:136).

En las descripciones presentadas en (1) pueden observarse características propias de la categoría de aspecto. Así en (1a) se destaca el valor puntual de la forma canté, esto es, se destaca su valor perfectivo. Sin embargo, bien es sabido que la puntualidad de una acción pasada también puede expresarse mediante la forma cantaba, tal como ha puesto de manifiesto Weinrich mediante el ejemplo: "El reloj daba la una cuando entró”. (1968:199). En (1b) y (1c) se recurre al valor aspectual iterativo, un valor asignado en el nivel elemental al imperfecto y en el nivel intermedio al indefinido, sin proporcionar mayor información que facilite la distinción entre los dos pretéritos. Por último, la definición propuesta en (1d) no está lejos de una equiparación entre "acción en desarrollo" y "acción durativa", acción

${ }^{1}$ El Plan curricular del Instituto Cervantes presenta una caracterización de las formas canté y cantaba a través de valores primarios y secundarios. En los primeros niveles la descripción se centra en los valores primarios, así la forma cantaba es definida a partir de su valor descriptivo y habitual, y la forma canté para designar "acciones pasadas enmarcadas en un momento temporal preciso" (PCIC A1-A2, Vol. I:130-131). Esta definición se mantiene para los niveles B1-B2 pero se añade una distinción semántica relacionada con la tipología verbal utilizada en pretérito perfecto simple; a su vez, se destaca la simultaneidad del pretérito imperfecto con una acción pasada (PCIC B1-B2, Vol. II: 75-76). Por otra parte, comienzan a introducirse algunos valores secundarios de la forma cantaba que terminan conformando una serie de usos que se extienden hasta los niveles superiores (PCIC C1-C2, Vol. III:76-77).

${ }^{2}$ No existe un consenso en los estudios gramaticales en cuanto a lo que representan los términos perfectivo e imperfectivo (García Fernández, 1998, 2013). Aquí nos interesa la lectura que realizan las gramáticas y manuales de ELE, en los que dichos términos son empleados para designar terminación o puntualidad de un evento, y duración o continuación de una acción. 
que también puede ser expresada mediante la forma canté, tal como se desprende de otro ejemplo formulado por Weinrich: "La guerra de los 100 años duró en realidad 116 años." (1968:199).

Estas aproximaciones no son exclusivas de los manuales de ELE editados en países hispanohablantes sino que también se encuentran en materiales creados por hispanistas coreanos y destinados a aprendientes coreanos. Así Lee en su la Introducción a la lingüística española (2006:115-123) plantea que la distinción entre el pretérito perfecto simple y el pretérito imperfecto se explica a partir de los morfemas aspectuales que componen a estos dos tiempos, morfemas que según este autor designan una acción verbal puntual y terminada o una acción que dura en el pasado.

Un tratamiento similar reciben las formas canté y cantaba en la Gramática del español para estudiantes universitarios de Kim y Kim. Los autores enumeran una serie de usos que se pueden resumir en los siguientes términos (2012:164-174):

La forma canté se utiliza para:

1. designar acciones puntuales,

2. expresar repetición en un período de tiempo cerrado,

3. enunciar la sucesión de eventos,

4. relatar una biografía.

La forma cantaba se utiliza para:

1. dar cuenta de una situación que duraba o continuaba en el pasado,

2. describir acontecimientos,

3. señalar hábitos que se repiten en el pasado,

4. describir a personas.

No es de extrañar que definiciones como las presentadas susciten confusiones entre los aprendientes de ELE, máxime si estas explicaciones son ilustradas mediante frases aisladas en las que no es posible percibir las funciones que las formas canté y cantaba desarrollan en un contexto discursivo de mayor complejidad (Gutiérrez Araus, 1996; Fernández, 1997; Güell, 2002) y por otra parte, mediante ejemplos en los que algunos valores atribuidos a los pretéritos perfecto simple e imperfecto no son propios de estos tiempos verbales, sino que son inherentes al lexema verbal de las formas utilizadas.

\section{FUNCIONES SIGNIFICATIVAS DE LAS FORMAS CANTÉ Y CANTABA EN EL DISCURSO INACTUAL}

En un intento por superar las deficiencias que suponen planteamientos didácticos como los que hemos reseñado en el apartado anterior, Gutiérrez Araus (1995, 1998, 2007) propone llevar a cabo una descripción de las funciones significativas de las formas canté 
y cantaba atendiendo a su distribución en el discurso inactual ${ }^{3}$. Para ello la autora destaca que, en primer lugar, la distinción entre las mencionadas formas ha de ser explicada a partir de la oposición entre acción principal frente a acción secundaria, simultánea a la principal. En segundo lugar, otro factor que se ha de tener en cuenta para realizar una descripción totalizadora de la oposición cantél cantaba es la distinción entre narración y descripción. En este sentido, hay que añadir que frente a la función narrativa de la forma canté, mediante la forma cantaba no solo se llevan a cabo descripciones sino que también se pueden expresar acciones habituales en el pasado.

Asimismo, en esta distribución interviene otro factor fundamental: el modo de acción verbal. Con verbos dinámicos el pretérito perfecto simple propicia la narración principal y con verbos de estado se indica los cambios de estado dentro de dicha narración. Por su parte, los verbos dinámicos en pretérito imperfecto dan lugar a la narración secundaria y de hechos repetidos como hábitos, y con los verbos estativos se lleva a cabo una descripción en el pasado, tal como puede observarse en el siguiente ejemplo:

\begin{abstract}
Tenía los pies y las manos helados y un hormigueo desagradable le recorría el costado izquierdo. Supo que se moría y le extrañó la precisión con que aún podía registrar detalles nimios. (Mendoza, La ciudad de los prodigios).
\end{abstract}

En la secuencia presentada en (2) el pretérito imperfecto tenía describe las circunstancias en las que se van a producir los cambios en la narración introducidos por un verbo estativo: la forma supo, que junto al verbo dinámico extrañó, conforman la narración principal. Las formas moría y podían expresan simultaneidad a las formas destinadas a la narración principal.

Teniendo en cuenta las características presentadas para las formas canté y cantaba en el discurso inactual, presentamos el estudio realizado en el que examinamos si las cinco funciones descritas para estas formas suponen el mismo grado de dificultad para su aprendizaje o si, por el contrario, dicha dificultad se ciñe a determinadas funciones.

\title{
4. EL ESTUDIO
}

Con el fin de señalar con precisión cuáles son los principales problemas que encuentran los aprendientes de ELE coreanos a la hora de narrar hechos pasados, hemos realizado un estudio en el que se examina la distribución que realizan de las formas canté y cantaba a través del discurso inactual.

\footnotetext{
${ }^{3}$ Benveniste pone de relieve que el concepto de tiempo no es suficiente para definir la posición que ocupa una forma dentro del sistema verbal, como tampoco lo es apelar a la morfología para dar cuenta de la organización de las formas verbales dentro de dicho sistema. Por esta razón propone analizar el comportamiento de las formas verbales atendiendo a dos planos de la enunciación: "Ces deux systèmes manifestent deux plans d'énonciation différent, que nous distinguerons comme celui de l'histoire et celui du discours" (1966:238). En el plano de la historia, también denominado inactual o del mundo narrado, los hechos enunciados corresponden a sucesos que tuvieron lugar en un determinado momento del pasado y son presentados sin intervención del enunciador.
} 


\subsection{Diseño}

Para la obtención de datos nos hemos servido de un ejercicio de huecos basado en una narración impersonal escrita. Esta narración fue elaborada a partir del texto "Las hermanas" utilizado en el proyecto de investigación SPLLOC 2 (Spanish Learner Language Oral Corpora $)^{4}$. La elección de este texto responde al diseño que presenta el mismo, el cual incorpora a lo largo del relato las cinco funciones significativas de las formas canté y cantaba en el discurso inactual. En cuanto a los verbos que componen esta narración impersonal y sobre los que el aprendiente debía elegir el tiempo verbal que creyera más adecuado, el texto se estructura en 25 núcleos verbales, los cuales a partir de su modo de acción son clasificados del siguiente modo:

Tabla 1. Tipos de verbos empleados en la prueba de obtención de datos

\begin{tabular}{|l|l|l|l|}
\hline \multicolumn{2}{|c|}{ Verbos estativos } & \multicolumn{2}{|c|}{ Verbos dinámicos } \\
\hline & visitar & leer & \\
ser & comer & pintar & despertarse \\
haber & beber & escribir & terminar \\
creer & hablar & ver & Ilegar \\
sentir & ayudar & ir & coger \\
necesitar & heírse & hacer & tranquilizarse \\
& jugar & acostarse & comer \\
\hline
\end{tabular}

La distribución de estos verbos a lo largo de la narración conforma las siguientes secuencias:

(3) a. Sarah y Gwen visitar la ciudad, comer tapas y beber vino. Después decidieron ir a Barcelona, coger el tren y hablar sobre su niñez.

b. De pequeñas, ser muy diferentes. Gwen, de niña, cada fin de semana leer un libro, escribir un cuento y pintar un cuadro. Durante la semana despertarse temprano y terminar sus deberes temprano. Sarah, de niña, los fines de semana jugar al fútbol y ver una película. Durante la semana ir al colegio en bicicleta y llegar tarde a clase. Hacer sus deberes por la noche y acostarse muy tarde.

c. De repente, en el tren haber un gran revuelo. Ellas creer que había un problema. Gwen sentir agua de lluvia en su cabeza. Entonces, necesitar la ayuda del revisor. El revisor las ayudar a cambiarse de asiento. Tranquilizarse.

d. Al final en Barcelona, comer una pizza y reírse al recordar el viaje.

${ }^{4}$ El proyecto al que hacemos referencia (RES-000-23-1609 y RES-062-23-1075) fue llevado a cabo por la Universidad de Southampton, la Universidad de Newcastle y la Universidad de York en el Reino Unido entre los años 2008 y 2010. Toda la información del proyecto SPLLOC2 puede encontrarse en http://www.splloc.soton.ac.uk/. 


\subsection{Participantes}

Los participantes de este estudio son estudiantes del departamento de español de la Universidad de Hankuk de Estudios Extranjeros. Para nuestra investigación seleccionamos estudiantes pertenecientes al segundo, tercero y cuarto año de la carrera de filología hispánica. Este criterio de clasificación se aplicó en la medida en que es a partir de estos cursos cuando comienza a ponerse en práctica la distinción de las formas canté y cantaba ${ }^{5}$. Por otra parte, en la selección de estos participantes se tuvo en cuenta que los estudiantes no fueran hablantes nativos o casi nativos del español ${ }^{6}$.

Por último, también quisimos incorporar los datos de aquellos estudiantes que, por haber realizado un intercambio con una universidad española o latinoamericana, no solo habían estado expuestos a la enseñanza del español como lengua extranjera sino que además habían recibido una instrucción en un contexto de aprendizaje del español como segunda lengua ${ }^{7}$. De esta manera, se seleccionó a un total de 60 participantes distribuidos en cuatro grupos de 15 participantes cada uno, tal como se indica en la tabla 2:

Tabla 2. Clasificación de los participantes de la investigación

\begin{tabular}{|c|c|c|c|}
\hline Año académico & Nivel de ELE & Grupo & Participantes \\
\hline 2 & A2 & 1 & Participantes $1-15$ \\
\hline 3 & B1 & 2 & Participantes $16-30$ \\
\hline 4 & B2 & 3 & Participantes $31-45$ \\
\hline 4 & B2 & 4 & Participantes $46-60$ \\
\hline
\end{tabular}

${ }^{5}$ La práctica de la distinción de estas dos formas se lleva a cabo en las clases de conversación de los niveles intermedio 1 y 2, y avanzado 1 y 2 . En cuanto a la instrucción formal, esta se realiza en los cursos de gramática de nivel elemental e intermedio atendiendo a los criterios presentados por Kim y Kim (2012) que hemos resumido en $\S 2$.

${ }^{6}$ En Corea se denomina a este tipo de estudiantes mediante el vocablo kyopo, el cual hace referencia a los coreanos que han nacido o han vivido la mayoría de su vida en un país extranjero. En términos históricos y por lo que interesa resaltar para esta investigación, a partir de la década del cincuenta del pasado siglo la diáspora de este colectivo registra un incremento en América Latina. En la actualidad muchos de los hijos de estos inmigrantes o descendientes de inmigrantes vuelven a Corea del Sur para realizar sus estudios universitarios.

${ }^{7}$ Por Lengua Extranjera (LE) se entiende aquella lengua no nativa que no está presente en el contexto donde es aprendida, es decir, la lengua que se aprende, no pertenece a la comunidad en la que vive el aprendiente (Pastor Cesteros, 2006:66). Baralo (2004:23) añade como característica a la LE el hecho de que esta lengua sólo es aprendida en un contexto institucional, ya sea un colegio, una universidad o una academia. En cuanto a la segunda lengua (L2), se trata de una lengua que es aprendida en el contexto inmediato del aprendiente, éste puede escucharla o hablarla también fuera del aula, es decir, se trata de una lengua que es adquirida en un contexto no sólo institucional sino también natural. 


\subsection{Procedimiento y análisis}

La aplicación de la prueba se llevó a cabo de forma individualizada, es decir, se entrevistó a cada participante de forma individual una única vez para obtener sus datos personales y evitar que la participación de los informantes fuera irregular. Durante la entrevista se les explicó a los participantes las instrucciones del ejercicio y se les otorgó diez minutos para realizarlo.

A la hora de interpretar los datos obtenidos nos hemos servido de la metodología del Análisis del Discurso ${ }^{8}$, análisis con el que hemos examinado la organización de la estructura temporal de las narraciones de los aprendientes coreanos, atendiendo a las funciones significativas de las formas canté y cantaba en el discurso inactual y señalando al mismo tiempo los contextos más problemáticos en el uso de los dos formas.

Por otra parte, debemos señalar que los resultados fueron analizados dentro del marco de los estudios cualitativos en los que la investigación se centra en describir, entender y clarificar un determinado fenómeno (Dörney: 2007:126). No obstante, también aportamos datos cuantitativos (cifras absolutas y porcentajes) desglosados en los cuatro grupos de participantes de nuestro estudio.

\section{DISCUSIÓN DE LOS RESULTADOS}

Los resultados obtenidos revelan que existe una tendencia generalizada en los cuatro grupos a utilizar las formas canté y cantaba en el discurso inactual. Sin embargo, también hemos registrado la presencia de otras formas verbales cuya distribución en los cuatro grupos de participantes presentamos en la tabla 3 .

Las cifras de la tabla 3 reflejan que la forma más utilizada es canté, seguida de la forma cantaba, con excepción del grupo 4 en el que se invierte el porcentaje de uso. En cuanto a las formas canto y cantaría, su representación no supone un problema generalizado en las producciones analizadas ya que se trata de casos aislados que responden a una confusión originada por la morfología verbal, es decir, se trata de errores formales y no funcionales. No se puede afirmar lo mismo de los once usos de la forma había cantado cuya utilización corresponde a la narración de un único participante, quien no interpreta el contenido temporal de la forma había cantado como la expresión de la anterioridad respecto a un momento anterior al momento de la enunciación, sino como la gramaticalización de la distancia al momento de la enunciación?

${ }^{8}$ El origen del Análisis del Discurso (AD) como sub-disciplina de la lingüística podemos encontrarlo en trabajos como el de Zellig Harris (1952) "Discourse analysis" donde se exponen los vínculos entre el texto y su situación social; en las investigaciones de Dell Hymes (1971) sobre la perspectiva sociológica del discurso en su entorno social y en la lingüística funcional con representantes como Michael Halliday (1985) y Talmy Givón (1986). Aunque no sería lícito afirmar que el AD es una disciplina homogénea, lo cierto es que en los postulados de los autores mencionados podemos encontrar similitudes en el interés por describir la lengua en uso, ya sea escrita u oral. Por lo que respecta a la aplicación del $\mathrm{AD}$ a los estudios sobre la adquisición de segundas lenguas, su interés se centra en el discurso entendido como una unidad de significado supraoracional, es decir, se centra en interacciones, conversaciones o textos (Larsen-Freeman y Long, 1994). Estos últimos conforman nuestra unidad de análisis.

${ }_{9} \mathrm{El}$ análisis de estos errores excede el objetivo del presente estudio por lo que no nos detendremos en ellos. 
Tabla 3. Morfología verbal utilizada en la prueba de obtención de datos

\begin{tabular}{|c|c|c|c|c|c|c|c|c|}
\hline \multirow{2}{*}{$\begin{array}{l}\text { Formas } \\
\text { verbales }\end{array}$} & \multicolumn{2}{|c|}{$\begin{array}{l}\text { Grupo } 1 \\
\text { (A2) }\end{array}$} & \multicolumn{2}{|c|}{$\begin{array}{c}\text { Grupo } 2 \\
\text { (B1) }\end{array}$} & \multicolumn{2}{|c|}{$\begin{array}{c}\text { Grupo } 3 \\
\text { (B2) }\end{array}$} & \multicolumn{2}{|c|}{$\begin{array}{c}\text { Grupo } 4 \\
(\text { B2+) }\end{array}$} \\
\hline & $\%$ & $(n)$ & $\%$ & $(n)$ & $\%$ & (n) & $\%$ & (n) \\
\hline Canté & 58.1 & (218) & 64 & (240) & 50.1 & (188) & 48.2 & (181) \\
\hline Cantaba & 40.8 & (153) & 32.5 & (122) & 49.6 & (186) & 51.4 & (193) \\
\hline Canto & 0.8 & (3) & 0.5 & (2) & 0 & (0) & 0 & (0) \\
\hline Había cantado & 0 & (0) & 2.9 & (11) & 0 & (0) & 0 & (0) \\
\hline Cantaría & 0.2 & (1) & 0 & (0) & 0.2 & (1) & 0.2 & (1) \\
\hline Total & & (375) & & (375) & & (375) & & (375) \\
\hline
\end{tabular}

Ahora bien, aunque las cifras de la tabla 3 reflejan una importante tendencia a utilizar las formas canté y cantaba en el discurso inactual, existen contextos en los que varios aprendientes han tenido dificultades a la hora de reconocer las funciones significativas de estas dos formas.

\subsection{Problemas en el reconocimiento de las funciones significativas de la forma canté}

Los problemas encontrados en la prueba de obtención de datos se originan principalmente por la falta de reconocimiento de las funciones que los verbos estativos en pretérito perfecto simple realizan en el discurso inactual, seguido en menor medida de los verbos dinámicos, tal como queda reflejado en la tabla 4:

Tabla 4. Problemas en el reconocimiento de las funciones significativas de la forma canté originados por la influencia del semantismo verbal

\begin{tabular}{|c|c|c|c|c|c|c|c|c|}
\hline \multirow{2}{*}{ Tipología verbal } & \multicolumn{2}{|c|}{$\begin{array}{c}\text { Grupo } 1 \\
\text { (A2) }\end{array}$} & \multicolumn{2}{|c|}{$\begin{array}{c}\text { Grupo } 2 \\
\text { (B1) }\end{array}$} & \multicolumn{2}{|c|}{$\begin{array}{c}\text { Grupo } 3 \\
\text { (B2) }\end{array}$} & \multicolumn{2}{|c|}{$\begin{array}{c}\text { Grupo } 4 \\
(\mathrm{~B} 2+)\end{array}$} \\
\hline & $\%$ & $(n)$ & $\%$ & $(n)$ & $\%$ & $(n)$ & $\%$ & $(n)$ \\
\hline $\begin{array}{l}\text { Confusiones con } \\
\text { verbos estativos }\end{array}$ & 1.8 & $(7 / 375)$ & 3.4 & $(13 / 375)$ & 2.1 & $(8 / 375)$ & 2.9 & $(11 / 375)$ \\
\hline $\begin{array}{l}\text { Confusiones con } \\
\text { verbos dinámicos }\end{array}$ & 0.8 & $(3 / 375)$ & 1.1 & $(4 / 375)$ & 0.2 & $(1 / 375)$ & 1.3 & $(5 / 375)$ \\
\hline Total de confusiones & 2.6 & $(10 / 375)$ & 4.5 & $(17 / 375)$ & 2.4 & $(9 / 375)$ & 4.2 & $(16 / 375)$ \\
\hline
\end{tabular}


Las cifras de la tabla 4 representan los casos en los que los aprendientes utilizan la forma cantaba por la forma canté desatendiendo a los cambios que se producen en la narración mediante los verbos estativos y enfocando algunas secuencias relevantes para el argumento de la narración como secundarias a través de los verbos dinámicos. En efecto, las confusiones que estamos analizando se producen porque los aprendientes no utilizan verbos estativos en pretérito perfecto simple en aquellos contextos en los que la narración exige un cambio de estado. Esta falta de reconocimiento tienen lugar con los verbos haber, sentir y necesitar, tal como puede observarse en los siguientes ejemplos:

(4) a. De repente, en el tren había un gran revuelo. (P4, P5, P6, P7, P8, P19, P20, P22, P25, P29, P34, P36, P39, P42, P46, P47, P50, P51, P52, P57, P59)

b. Gwen sentía agua de lluvia en su cabeza. (P16, P21, P22, P26, P34, P35)

c. Entonces, necesitaban la ayuda del revisor, quien las ayudó a cambiarse de asiento. (P5, P17, P36, P51, P55, P56, P60).

Los ejemplos de (4) destacan que la forma más conflictiva para los cuatro grupos de estudiantes coreanos es la forma había, seguida de sentía y necesitaban. Los aprendientes atribuyen un valor descriptivo a estas secuencias sin tener en cuenta el cambio que se produce en el argumento de la narración y propiciando una lectura de simultaneidad en detrimento de la sucesión de los eventos del relato.

La aparición de la forma cantaba con verbos dinámicos en contextos en los que prima la secuencia narrativa de eventos narrados en sucesión se produce en un número más reducido de narraciones:

(5) a. En el verano de 2006 Sarah y Gwen fueron a España. En Madrid visitaron la ciudad, comían tapas y bebían vino. (P15, P51)

b. Después decidieron ir a Barcelona, cogían el tren y hablaron sobre su niñez. (P50).

c. Entonces, necesitaron la ayuda del revisor, quien las ayudaba a cambiarse de asiento. (P24)

d. Una vez en su nuevo asiento, se tranquilizaban. (P28, P29, P34)

e. Al final en Barcelona, comían una pizza y se rieron al recordar el viaje. (P51)

En el ejemplo (5a) el uso de las formas comían y bebían no puede ser interpretado como la expresión de hechos repetidos como hábitos en el pasado debido a la inexistencia de algún marcador temporal que propicie dicha lectura. Por el contrario, se trata de un pasaje en el que prima la secuencia narrativa de eventos presentados en sucesión, es decir, no tiene lugar la expresión de la simultaneidad respecto a un momento anterior a la enunciación que caracteriza a las formas mencionadas. Esta explicación también se aplica al uso de cogían, ayudaba, se tranquilizaba y comían de los ejemplos (5b-e). 
Los ejemplos de (4) y (5) ponen de manifiesto que la falta de reconocimiento de las funciones de la forma canté en el discurso inactual atraviesa la interlengua de aprendientes coreanos pertenecientes a cuatro niveles de competencia comunicativa diferente, siendo el reconocimiento de los cambios de estado en una narración, la función que más dificultades ocasiona para los aprendientes en cuestión. A su vez, el aumento en el porcentaje de estas confusiones a medida que se desarrolla la interlengua de los aprendientes indica que se trata de un contexto complejo que requiere una instrucción específica.

\subsection{Problemas en el reconocimiento de las funciones significativas de la forma cantaba}

Los datos analizados revelan que el reconocimiento de las funciones de la forma cantaba en el discurso inactual resulta más complejo para los participantes de nuestro estudio que las funciones de la forma canté, tal como puede apreciarse en las cifras de la tabla 5:

Tabla 5. Problemas en el reconocimiento de las funciones significativas de la forma cantaba originados por la influencia del semantismo verbal

\begin{tabular}{|c|c|c|c|c|c|c|c|c|}
\hline \multirow{2}{*}{ Tipología verbal } & \multicolumn{2}{|c|}{ Grupo 1 (A2) } & \multicolumn{2}{|c|}{ Grupo 2 (B1) } & \multicolumn{2}{|c|}{ Grupo 3 (B2) } & \multicolumn{2}{|c|}{ Grupo 4 (B2+) } \\
\hline & $\%$ & (n) & $\%$ & (n) & $\%$ & (n) & $\%$ & (n) \\
\hline $\begin{array}{l}\text { Confusiones con verbos } \\
\text { dinámicos }\end{array}$ & 9.6 & $(36 / 375)$ & 15.5 & $(58 / 375)$ & 2.9 & $(11 / 375)$ & 1.6 & $(6 / 375)$ \\
\hline $\begin{array}{l}\text { Confusiones con verbos } \\
\text { estativos }\end{array}$ & 0.8 & $(3 / 375)$ & 2.1 & $(8 / 375)$ & 1.1 & $(4 / 375)$ & 0.2 & $(1 / 375)$ \\
\hline Total de confusiones & 10.4 & $(39 / 375)$ & 17.6 & $(66 / 375)$ & 4 & $(15 / 375)$ & 1.8 & $(7 / 375)$ \\
\hline
\end{tabular}

A diferencia de lo que ocurre con los problemas suscitados en las funciones de la forma canté, donde el porcentaje de confusiones no se reduce en los niveles más avanzados de ELE, con la forma cantaba el número de confusiones disminuye a medida que se incrementa el conocimiento de la lengua meta. Otra diferencia significativa es que las dificultades en el reconocimiento de la forma cantaba se concentran en los verbos dinámicos, lo que se traduce en una falta del reconocimiento del uso de esta forma para la narración de hechos repetidos como hábitos, como en:

(6) a. Gwen, de niña, cada fin de semana leía un libro, escribía un cuento y pintaba un cuadro. Durante la semana se despertó temprano y terminó sus deberes temprano. (P15, P21, P26, P32, P48)

b. Durante la semana iba al colegio en bicicleta y llegó tarde a clase (P4, P15).

c. Sarah, de niña, los fines de semana jugaba al fútbol y veía una película. [...] Hacía sus deberes por la noche y se acostó muy tarde. (P21, P26, P48) 
Los ejemplos (6a-c) reflejan que los aprendientes trasladan las propiedades del lexema verbal de formas como se despertó, terminó, llegó y se acostó, formas caracterizadas por el rasgo perfectivo, al uso de la morfología verbal en un contexto en el que las acciones son presentadas como hábitos en el pasado. No es de extrañar que esto suceda ya que, como hemos mencionado más arriba, estos aprendientes han estado expuestos a una enseñanza en la que la distinción cantél cantaba es presentada en términos aspectualistas en tanto que formas perfectivas e imperfectivas, respectivamente.

\section{CONCLUSIONES}

Los datos obtenidos en nuestro estudio revelan que no todas las funciones significativas de las formas canté y cantaba representan el mismo grado de complejidad para los aprendientes coreanos. Mientras que con el pretérito perfecto simple el número de confusiones es mayor con los verbos estativos dando lugar a una falta de reconocimiento de los cambios de estado que se producen en una narración, con el pretérito imperfecto las confusiones se concentran en los verbos dinámicos, especialmente con verbos perfectivos, ocasionando problemas en la narración de hábitos en el pasado.

Otro dato significativo es que a diferencia de lo que ocurre con los verbos dinámicos en pretérito imperfecto, en los que el número de confusiones disminuyen a medida que aumenta el nivel de ELE, las confusiones de la interacción del perfecto simple con verbos estativos persisten en los niveles más avanzados, con el riesgo de llegar a fosilizarse en la interlengua de los aprendientes. Como hemos visto, estos problemas parecen estar inducidos no solo por la distancia entre la lengua materna de los aprendientes y la lengua meta, sino también por una didáctica centrada en destacar unas características que no siempre resultan adecuadas para describir el funcionamiento de las formas canté y cantaba en el paradigma verbal español.

A la luz de estas reflexiones parece necesario insistir en una instrucción que tenga en cuenta las cinco funciones que los pretéritos perfecto simple e imperfecto de indicativo representan en el discurso inactual. Por otra parte, en investigaciones futuras, debemos extender nuestro campo de análisis hacia otras actividades comunicativas, como la expresión oral o la comprensión auditiva, para contrastar los resultados aquí expuestos con los de nuevas pruebas en las que los aprendientes no dispongan de tiempo para monitorizar su producción. Asimismo, estas pruebas habrán de estar diseñadas en torno a ejercicios de expresión libre para poder comparar qué uso hacen los aprendientes coreanos de la morfología verbal en el discurso inactual cuando no son sometidos a una narración controlada como la realizada para este estudio.

\section{REFERENCIAS BIBLIOGRÁFICAS}

AA. VV. (2006): Plan curricular del Instituto Cervantes: niveles de referencia para el español, Madrid: Biblioteca Nueva.

Baralo, M. (2004): La adquisición del español como lengua extranjera, Madrid: Arco/Libros.

Benveniste, E. (1966): Problèmes de linguistique générale, Paris: Gallimard. 
Сhoo, M. у Kwak, H. Y. (2008): Using Korean. A guide to contemporary usage, Cambridge: Cambridge University Press.

DöRnEY, Z. (2007): Research Methods in Applied Linguistics, Oxford: Oxford University Press.

FERNÁNDEZ, S. (1997): Interlengua y análisis de errores en el aprendizaje del español como lengua extranjera, Madrid: Edelsa.

GARCía Fernández, L. (1998): El aspecto gramatical en la conjugación, Madrid: Arco/Libros.

- (2013): El tiempo en la gramática, Madrid: Arco/Libros.

GüELL, L. (2002): “Análisis del contraste de los pasados y algunas propuestas didácticas”, MarcoELE, 10, 89-107. Disponible en red: http://marcoele.com/descargas/expolingua_2002.guell.pdf.

Gutiérrez Araus, M. (1995): Formas del pasado en Indicativo, Madrid: Arco/ Libros.

- (1996): "Problemas de la enseñanza a anglohablantes de dos formas de indicativo: imperfecto e indefinido", en S. Montesa y P. Gomis: Tendencias actuales en la enseñanza del español como lengua extranjera, 1, ASELE, Málaga, 33-41.

- (1998): "Sistema y discurso en las formas verbales del pasado", Revista española de Lingüística, $28,2,275-300$.

Gutiérrez Araus, M. (2007): Problemas fundamentales de la enseñanza del español como 2/L, Madrid: Arco Libros.

Givón, T. (1985): Syntax. A Functional Typological Introduction, vol. 1, Amsterdam: John Benjamins. Halliday, M.A.K. (1985): An Introduction to Functional Grammar, London: Edward Arnold.

HARRIS, Z. (1952): "Discourse Analysis", Language, 28 (1), 1 -30.

Hymes, D. H. (1971/1995): “Acerca de la competencia comunicativa”, en Llobera, M. (Ed.) Competencia Comunicativa, Madrid: Edelsa.

KIM, K. y Kim, H. S. (2012): Gramática del español para estudiantes universitarios, Seúl: HUINE.

LaRSEN-Freeman, D. y Long, M. H. (1994): Introducción al estudio de segundas lenguas, Madrid: Gredos.

LEE, M. K. (2006): 스페인어학의 이해 (Introducción a la lingüística española), 서울: 한국문화사.

LEE, M. K. y Son, J. E. (2009): "La difusión del español en Corea, y la interferencia del coreano y del inglés en el aprendizaje del español", comunicación presentada en el III Congreso internacional de FIAPE: La enseñanza del español en tiempos de crisis. Cádiz. pp. 1-19. Disponible en red: http:// www.mecd.gob.es/redele/Biblioteca-Virtual/2010/Numeros-Especiales/FiapeIIICongreso.html.

Long, A. y Whatley, M. (2014): "Investigating the role of learners' first language in the acquisition of past-time expression: A study of Korean-speaking learners of Spanish", comunicación presentada en el Congreso de la American Association for Applied Linguistics, Portland: en prensa.

Pastor Cesteros, S. (2006): Aprendizaje de segundas lenguas. Lingüística aplicada a la enseñanza de idiomas, Alicante: Publicaciones de la Universidad de Alicante.

WeINRICH, H. (1968): Estructura y función de los tiempos en el lenguaje, Madrid: Gredos. 
